\title{
Statistically Deformable Face Models for Cranio-Facial Reconstruction
}

\author{
Peter Claes ${ }^{1}$, Dirk Vandermeulen ${ }^{1}$, Sven De Greef ${ }^{2}$, Guy Willems ${ }^{2}$ \\ and Paul Suetens ${ }^{1}$ \\ ${ }^{1}$ Katholieke Universiteit Leuven, Medical Image Computing - ESAT/PSI, Belgium \\ ${ }^{2}$ Katholieke Universiteit Leuven, Centre of Forensic Ontology, Belgium
}

Forensic facial reconstruction aims at estimating the facial outlook associated to an unknown skull specimen. Estimation is based on tabulated average values of soft tissue thicknesses measured at a sparse set of landmarks on the skull. Traditional 'plastic' methods apply modeling clay or plasticine on a cast of the skull approximating the estimated tissue depths at the landmarks and interpolating in between. Current computerized techniques mimic this landmark interpolation procedure using a single facial surface template. However, the resulting reconstruction is biased by the specific choice of the template and no face-specific regularization is present. We reduce the bias by using a flexible statistical model of a dense set of facial surface points combined with an associated sparse set of skull landmarks. The statistical model also provides a probability value, which can be used to regularize the reconstruction towards more plausible outlooks. The reconstruction is obtained by fitting the model skull landmarks to the corresponding landmarks indicated on a digital copy of the skull to be reconstructed. The fitting process alternates between changing the face-specific statistical model parameters and interpolating the remaining landmark fit error using a minimal bending Thin-Plate Spline (TPS)-based deformation. Furthermore, estimated properties of the skull specimen (BMI, age and gender e.g.) can be incorporated as conditions on the reconstruction by removing property-related shape variation from the statistical model description before the fitting process. This iterative statistical model-based reconstruction process is shown by experiment to converge to a realistic reconstruction of the face, independent of the initial template.

Keywords: computer-aided, cranio-facial reconstruction, statistical models, thin-plate splines.

\section{Introduction}

When confronted with a corpse that is unrecognizable due to its state of decomposition, skeletonisation, mutilation or incineration, and if no other identification evidence is available, craniofacial reconstruction can be considered. The goal of craniofacial reconstruction is to recreate an estimate of the face of an individual at the time of death. Hopefully, this will trigger recognition by relatives such that further identification evidence can be gathered on a restricted list of candidates. Although craniofacial reconstruction is a valuable tool in the initiation of the process of identification, positive identification has to be obtained eventually by classic techniques such as radiographic and dental comparisons or DNA-analysis.

Several 3D manual methods for facial reconstruction have been developed and are currently used in practice. These reconstructions consist of physically modeling a face on a skull replica (the target skull) with clay or plasticine. However, manual reconstruction methods require a lot of anatomical and artistic modeling expertise and are, as a result, highly subjective. Furthermore, these reconstructions take a lot of time (several days), and, hence, are often limited to a single reconstruction. Computer-based methods, on the other hand, are consistent (given the same input data and modeling assumptions, the same output results) and objective (knowing all the modeling assumptions). Moreover, since these methods can be executed in a short time, multiple reconstructions from the same skull using different modeling assumptions (older, thicker, ...) can be obtained.

Current computerized reconstruction techniques are limited, though, in the model used for reconstructing the complete facial outlook. First, either a generic face template or a specific best 
look-alike template, based on skull similarities or properties (BMI, gender and age e.g.), is chosen. Subsequently, the skin surface associated to the target skull is estimated by deforming the model template, based on a generic, "smooth" interpolation of a deformation that maps corresponding landmark points of the model skull on the target skull. Multiple reconstructions based on different values of BMI, age and gender are obtained by choosing a different starting face template. Two major shortcomings are apparent using such a static model in combination with a generic deformation. First, the reconstruction can be incorrectly biased by the choice of the template. Indeed, when using a subject-specific best look-alike template based on similarity in ancestry, gender and age, unwanted facial features of the template remain visible in the final reconstruction. Using a generic face template, on the other hand, results in a too smooth and unspecific reconstruction. Secondly, the generic deformations applied are not face-specific, they are just "smooth". No problem arises when the differences between the model and target skull landmarks are small. However, when these differences are relatively large, the required deformation will be more pronounced, which can result in unrealistic, caricature-like or implausible facial reconstructions.

Current computer-based facial reconstruction methods differ mainly by the selection of landmark points or skull features used to deform the model towards a given target skull. Some techniques $[1,2,3,4,5,14]$ fit a facial template to the endpoints of a set of virtual dowels positioned on a 3D digitized model of the target skull. The dowel lengths represent averages of ancestry-, gender- and age-matched tissue depths at a limited number of predefined cephalometric landmarks. There is no direct correlation, however, between the reported tissue depths and the associated skin surface shape of an individual. Other computer-based techniques deform a 3D reference skull to a target skull based on crest lines (lines of maximal local curvature) [6], control data sets [7] or feature points [8]. The calculated skull deformation is then extrapolated and applied to the skin surface associated to the reference skull. A reference skull is selected based on similarity in ancestry, gender and age. Reference skulls and corresponding facial surfaces are obtained using CT scanning, which limits the selection of the reference database to patient data because of the involved irradiation dose.
In order to eliminate the template-related bias and to minimize the unrealistic character of the reconstructions caused by large model deformations, we propose a new flexible facial model for craniofacial reconstruction, modeling the combined population-dependent variation and correlation of skin surface shape and tissue depth (represented by skull landmarks), calculated from a facial database. This template can be considered as an elastic mask with elastic dowels at particular locations on the inside of the mask. The elasticity of the mask and the dowels is defined as the statistically allowed correlated variation of facial surfaces and tissue depths learned from the database.

A first scenario for reconstruction could be to use the average template (face plus skull landmarks) as an initial mask and to fit it to the target skull landmarks. By changing the statistical model parameters between the statistically determined boundaries, the mask is deformed in a face-specific way only. However, when the number of degrees of freedom in the statistical model is too small, faces atypical of the database will be poorly approximated this way with a resulting high fit error.

Instead, we augment the statistical model with an additional smooth, generic deformation model, such that the deformation of the statistical model interpolates the skull landmarks. This deformation is modeled by a Thin-Plate-Spline (TPS) function, mapping corresponding model and target skull landmarks and smoothly extending the deformation in between. By alternating the statistical deformation and generic interpolation steps, a more plausible or realistic face is reconstructed without a bias towards a specific face in the database and with minimal deviation from the best approximating statistical face model. Multiple reconstructions based on different values of skull properties are obtained by modeling and removing facial variations originating from property differences in the facial database. Besides facial surface points and skull points, a numeric set of property values for every entry in the database is stored and incorporated during statistical model building. Before the fitting or reconstruction process, shape variation in the statistical model coded by the values of the given properties is removed. This results in a database of facial entries with the same property values as the skull specimen, on which a new statistical model with eliminated property variation can be calculated and used during reconstruction. 
The remainder of this paper is organized as follows. In section 2 we define the mathematical representation of the facial template used throughout the paper and explain the TPS interpolation machinery for facial reconstruction based on a static model. In section 3 the construction of the statistical facial model is described, while section 4 explains the explicit modeling of property-dependent facial variations. Section 5 will show how the statistical model can be combined with the TPS deformation for facial approximation. In section 6 a comparison is made between a reconstruction based on static models and a reconstruction based on our proposed flexible statistical model for a real skull case. Finally, in section 7, conclusions are drawn and some future improvements are listed.

\section{TPS-based Static Facial Model Fitting}

Our facial template for craniofacial reconstruction consists of a combination of two sets of 3D point coordinates $M=\left(M^{s}, M^{l m}\right)$, with $M^{s}=\left\{M_{j}^{s} \in R^{3} \mid j=1, \ldots, N\right\}$ a dense point set representing the facial surface of the model, and $M^{l m}=\left\{M_{i}^{l m} \in R^{3} \mid i=1, \ldots, L\right\}$ representing the set of model landmark or feature points (skull landmarks, thickness dowel ends, e.g), where typically $N>>L$. In this paper we will make use of anatomical landmarks defined on the skull surface.

The craniofacial reconstruction of a face $F$ now consists of estimating the dense set of facial surface point coordinates $F^{s}$ based on a model $M$ and of a set of corresponding landmark points $\left(F_{i}^{l m}, M_{i}^{l m}\right)$ on the model and target skull. One way to estimate $F^{s}$ is to determine a smooth mapping or deformation function $\mathbf{f}: R^{3} \rightarrow R^{3}$ satisfying the following interpolation conditions at the landmark points:

$$
F_{i}^{l m}=f\left(M_{i}^{l m}\right) \quad \text { for } \quad i=1, \ldots, L
$$

and simultaneously minimizing a deformation penalty $E_{\mathbf{f}}$ which is the sum of a componentwise bending energy functional $E_{f_{c}}$, with $f_{c}$ corresponding to the $x, y$ and $z$ components of the deformation function $\mathbf{f}$, resp.:

$$
\begin{gathered}
E_{f_{c}}=\int_{R^{3}}\left(\frac{\partial^{2} f_{c}}{\partial x^{2}}\right)^{2}+\left(\frac{\partial^{2} f_{c}}{\partial y^{2}}\right)^{2}+\left(\frac{\partial^{2} f_{c}}{\partial z^{2}}\right)^{2}+\ldots \\
2\left(\frac{\partial^{2} f_{c}}{\partial x \partial y}\right)^{2}+2\left(\frac{\partial^{2} f_{c}}{\partial x \partial z}\right)^{2}+2\left(\frac{\partial^{2} f_{c}}{\partial y \partial z}\right)^{2} d \mathbf{x}
\end{gathered}
$$

Thin-Plate Spline (TPS) functions, also known as smoothest interpolators, satisfy the conditions in (1) while minimizing the energy defined in (2). The null space of the penalty functionals $E_{f_{c}}$ are the linear (affine) transformations. Hence, the TPS functions interpolate the given conditions (1) while remaining as affine as possible, as measured by (2).Using homogeneous coordinate notations for $M^{l m}$ and $F^{l m}$, the TPSbased interpolation function can be specified by two parameter matrices $\mathbf{d}$ and $\Lambda$ :

$$
\mathbf{f}\left(M_{i}^{l m}\right)=M_{i}^{l m} \mathbf{d}+\phi\left(M_{i}^{l m}\right) \boldsymbol{\Lambda}
$$

where $\mathbf{d}$ is a $4 \times 4$ affine transformation matrix and $\Lambda$ is a $L \times 4$ non-affine warping coefficient matrix. The $1 \times L$ vector $\phi\left(M_{i}^{l m}\right)$ is related to the thin-plate spline $L \times L$ kernel matrix $\Phi$ with component values $\phi_{l}\left(M_{i}^{l m}\right)=\left\|M_{i}^{l m}-M_{l}^{l m}\right\|$. For a function of the form (3) the total bending energy can be rewritten as:

$E_{f}=\operatorname{trace}\left(\Lambda^{T} \Phi \boldsymbol{\Lambda}\right)$ with $\Phi_{i l}=\left\|M_{i}^{l m}-M_{l}^{l m}\right\|$

The TPS parameters $\mathbf{d}$ and $\boldsymbol{\Lambda}$ can be computed by solving the following linear equation:

$$
\left(\begin{array}{cc}
\Phi & M^{l m} \\
M^{l m^{T}} & 0
\end{array}\right)\left(\begin{array}{c}
\boldsymbol{\Lambda} \\
\mathbf{d}
\end{array}\right)=\left(\begin{array}{c}
F^{l m} \\
0
\end{array}\right)
$$

Once the TPS parameters are determined, an estimation $F_{e s t}^{s}$ of the facial surface points $F^{s}$ can be obtained by simple substitution of the model surface points into (3):

$$
F_{e s t}^{s}=M^{s} \mathbf{d}+\phi\left(M^{s}\right) \boldsymbol{\Lambda}
$$

From equation (4) we observe that the final minimum bending energy or the amount of component-wise non-affine deformation required to satisfy the interpolation conditions in Eq.(1) depends on the model landmark points $M^{l m}$. When the "difference" (except for an affine transformation) between the landmark points $M^{l m}$ and $F^{l m}$ increases, the amount of non-affine deformation will increase as well, and, as a result, the less face-like the estimation $F_{e s t}^{s}$ will 
be. Hence, the choice of model template is important. In the next section we will describe a statistical model that is capable of reducing the difference between $M^{l m}$ and $F^{l m}$ or the complete faces $M$ and $F$ in a face-specific way.

\section{PCA-based Statistical Facial Modeling and Fitting}

The flexible statistical facial template that we propose in this paper, models the combined population-dependent variation and correlation of skin surface shape and tissue depths (represented by skull landmarks) from a facial database of, currently, 118 faces. A single facial database entry consists of a dense point representation of the 3D facial surface (acquired with a 3D camera. Shapecam, Eyetronics), 52 skull landmarks and the nose tip as extra (skin) landmark. Skull landmarks are derived from soft tissue depths measured at 52 anatomical landmarks on the skin surface with an ultrasound device (Epoch4B, Panametrics) according to [13]. The soft tissue depths are set out starting from the landmarks on the skin surface perpendicular to the skin surface, resulting in 52 skull landmarks. Inter-subject correspondences between skull landmarks and dense skin surface points are automatically calculated using a non-linear robust point matching procedure for landmark correspondences [9] followed by a geodesic surface matching algorithm [10]. This results in consistent dens point representations of the $3 \mathrm{D}$ facial surfaces.

Using this procedure, we construct a database of faces $\left\{F_{k} \mid k=1, \ldots, K\right\}$ with $F_{k}=\left(X_{1}^{s}, Y_{1}^{s}\right.$, $Z_{1}^{s}, \ldots, X_{N}^{s}, Y_{N}^{s}, Z_{N}^{s}, X_{1}^{l m}, Y_{1}^{l m}, Z_{1}^{l m}, \ldots \quad, X_{L}^{l m}$, $\left.Y_{L}^{l m}, Z_{L}^{l m}\right)$ a vectorial representation of a single face, each coordinate index labeling corresponding points. New acceptable faces can then be expressed as linear combinations of the faces in the database:

$$
M(\mathbf{a})=\sum_{k=1}^{K} a_{k} F_{k}
$$

with reasonable limits on the range of the coefficient values $a_{k}$. A flexible facial model can then be defined as the set of faces $M(\mathbf{a})=$
$\left(M^{s}(\mathbf{a}), M^{l m}(\mathbf{a})\right)$ parameterized by the coefficients $\mathbf{a}=\left(a_{1}, \ldots, a_{K}\right)$.

A probability distribution can be associated to this linear face space by fitting a multivariate normal distribution to the data set of 118 faces. This can be accomplished using a Principal Component Analysis (PCA) of the covariance matrix of the mean normalized faces $\widehat{F}_{k}=F_{k}-\bar{F}$ with $\bar{F}=\frac{1}{K} \sum_{k=1}^{K} F_{k}$ the average face (shown in figure 1 (b)). PCA performs essentially a basis transformation to an orthogonal coordinate system (eigenspace) spanned by the eigenvectors $U_{k}$ of the covariance matrix in descending order according to their associated eigenvalues $\sigma_{k}$.

$$
M(\mathbf{c})=\bar{F}+\sum_{k=1}^{K-1} c_{k} U_{k}
$$

The flexible statistical facial model is then defined as the set of faces $M(\mathbf{c})$ parameterized by the coefficients $\mathbf{c}=\left(c_{1}, \ldots, c_{K-1}\right)$. The probability for the coefficients $\mathbf{c}$ generating a plausible face is given by:

$$
p(\mathbf{c}) \sim \exp \left[-\frac{1}{2} \sum_{k=1}^{K-1}\left(\frac{c_{k}}{\sigma_{k}}\right)^{2}\right]
$$

This prior probability can be used in a bayesian estimation framework to fit the model $M(\mathbf{c})$ to a new observed facial instance $F$ by maximizing the following a posteriori probability:

$$
P(\mathbf{c} \mid F) \sim P(F \mid \mathbf{c}) \cdot p(\mathbf{c}),
$$

or, equivalently, minimizing the following functional:

$$
E_{F, M(\mathbf{c})}(\mathbf{c})=\|F-M(\mathbf{c})\|^{2}+\sum_{k=1}^{K-1}\left(\frac{c_{k}}{\sigma_{k}}\right)^{2}
$$

For further details about optimizing (10) or (11) over the model parameters $(\mathbf{c})$, we refer the reader to [11].

The key point in using a flexible statistical model for facial reconstruction is the ability to update the facial template $M(\mathbf{c})$ by changing the coefficients $\mathbf{c}$ in a face-specific way such that no bias towards a specific face in the 


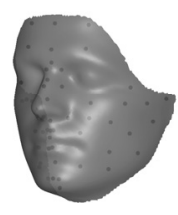

(a)

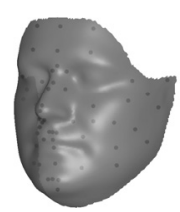

(d)

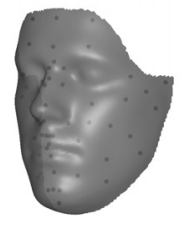

(f)

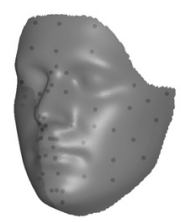

(b)

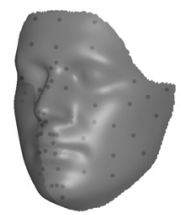

(b)

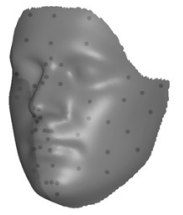

(b)

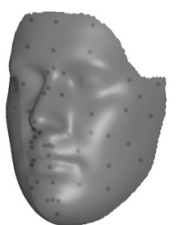

(c)

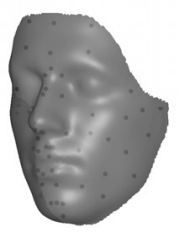

(e)

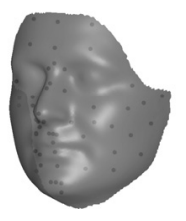

(g)
Fig. 1. (b)The average facial surface and skull landmarks (black dots) of our database. (a, c) visualize the effect of changing the first model parameter in the negative (a) and positive (c) direction of the first eigenvector. $(\mathrm{d}, \mathrm{e})$ and $(\mathrm{f}, \mathrm{g})$ visualize the effect according to the second and third eigenvector respectively.

database is introduced and such that the differences between the landmark points $M^{l m}(\mathbf{c})$ and $F^{l m}$ or the complete faces $M(\mathbf{c})$ and $F$ can be reduced, resulting in a smaller amount of remaining non-affine deformation required. Figure 1 (a,c-g) shows the effect of changing the first three model parameters of the average face. In section 5, we show how we can combine both models (the statistical PCA-based face deformation model presented in this section and the TPS-based generic, smooth deformation model presented in section 2) to arrive at realistic reconstructions of faces from skulls, even when the statistical face deformation model is too restrictive. But, first we show in the next section how face-specific property-dependent deformations can be extracted from the statistical model by incorporating property values of the facial entries in the database during model construction.

\section{Modeling Property-dependent Variations}

In order to model and extract property-dependent variations from the database the vector representations of the facial entries, defined in the previous section, need to be expanded. A numeric set of property values is appended at the end of every facial vector representation resulting into $F_{k}=\left(X_{1}^{s}, Y_{1}^{s}, Z_{1}^{s}, \ldots, X_{N}^{s}, Y_{N}^{s}, Z_{N}^{s}, X_{1}^{l m}\right.$, $\left.Y_{1}^{l m}, Z_{1}^{l m}, \ldots, X_{M}^{l m}, Y_{M}^{l m}, Z_{M}^{l m}, B, A, G\right)$ for every face $\left\{F_{k} \mid k=1, \ldots, K\right\}$ in the database. Both $B(b m i)$ and $A(a g e)$ have continuous values, while the $G$ (gender) values are discrete $(-1$ for females and +1 for males). Based on these vector representations, a statistical PCA template, modeling the combined skin surface shape, skull landmarks and properties variation, is built according to the previous section.

Expressing the original data in terms of principal components (equation 8) is in fact a description of the data in terms of variances. The goal is to find proper directions in this variance space related to variations in $A, B$ and $G$. The extraction of property-dependent variations from the statistical model is obtained by finding three basis vectors in the coordinate system spanned by the eigenvectors, describing the separate independent change of $B, A$ and $G$. Since this can be achieved by an infinite number of linear combinations of the eigenmodes, the linear combination which has the smallest Mahalanobisdistance $D$ to the origin of the eigenspace, is searched for. According to [11] this can be solved by a constrained least-square optimization. Let $\mathbf{r}_{b}, \mathbf{r}_{a}$ and $\mathbf{r}_{g}$ be the three unknown basis vectors describing a unit change of $B, A$ and $G$ respectively. The Mahalanobis-distance $D$ of these three vectors is given by:

$$
D\left(\mathbf{r}_{x}\right)=\sum_{k=1}^{K-1} \frac{\left(r_{x k}\right)^{2}}{\sigma_{k}} \text { for } x \in\{b, a, g\}
$$

Taking into account that $B, A$ and $G$ values depend only on the last three rows of the eigenvector matrix $U$, a sub-matrix $U^{s}$ consisting of the last three rows of $U$ can be defined. Minimization of the Mahalanobis-distance $D$ subject to the constraint of separate $B, A$ and $G$ change by one unit is done by minimizing the following Lagrange functional $L$ : 


$$
\begin{aligned}
& L\left(\mathbf{r}_{x}, \mathbf{l}_{x}\right)=\sum_{k=1}^{K-1} \frac{\left(r_{x k}\right)^{2}}{\sigma_{k}}-\mathbf{l}_{x}^{T}\left[U^{S} \mathbf{r}_{x}-\mathbf{e}_{x}\right] \\
& x \in\{b, a, g\} \\
& \mathbf{e}_{b}=\left[\begin{array}{l}
1 \\
0 \\
0
\end{array}\right], \mathbf{e}_{a}=\left[\begin{array}{l}
0 \\
1 \\
0
\end{array}\right], \mathbf{e}_{g}=\left[\begin{array}{l}
0 \\
0 \\
1
\end{array}\right]
\end{aligned}
$$

The vectors $\mathbf{I}_{x}$ for $x \in\{b, a, g\}$ are the Lagrange multipliers. The Lagrange functional $L\left(\mathbf{r}_{x}, \mathbf{l}_{x}\right)$ is solved by calculating the derivatives with respect to all the variables $\mathbf{l}_{x}$ and $\mathbf{r}_{x}$, resulting into the following two linear equations:

$$
\begin{aligned}
& 2 A^{-1} R=U^{s T} Q \\
& U^{s} R=I
\end{aligned}
$$

where,

$$
\begin{aligned}
A & =\left[\begin{array}{lll}
\sigma_{1} & & \\
& \ddots & \\
& & \sigma_{K-1}
\end{array}\right] \\
R & =\left[\begin{array}{lll}
\mathbf{r}_{b} & \mathbf{r}_{a} & \mathbf{r}_{g}
\end{array}\right] \quad Q=\left[\begin{array}{lll}
\mathbf{l}_{b} & \mathbf{l}_{a} & \mathbf{l}_{g}
\end{array}\right]
\end{aligned}
$$

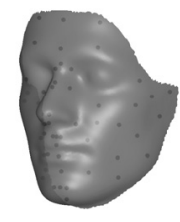

(a)

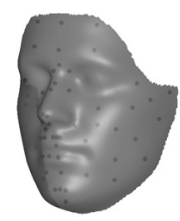

(d)

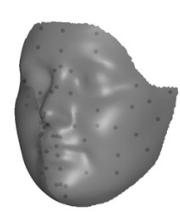

(d)

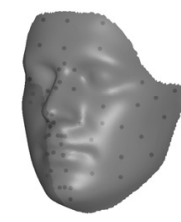

(b)

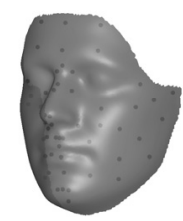

(b)

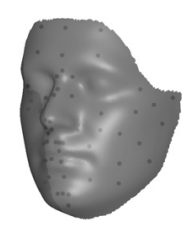

(b)

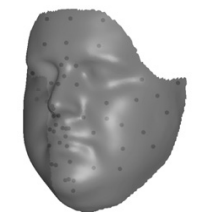

(c)

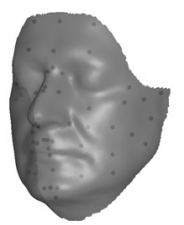

(e)

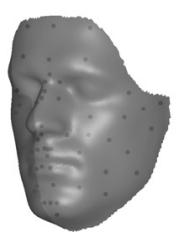

(e)
Fig. 2. (b)The average facial surface and skull landmarks (black dots) of our database with BMI $=$ 22.35, Age $=25$ and gender $=0.18$. (a) BMI changed to 15 , (c) BMI changed to 35, (d) Age changed to 18, (e) Age changed to 70, (f) Gender changed to -1 and (g) Gender changed to +1 .
The three basis vectors in $R$ are then given by:

$$
R=A U^{s T}\left[U^{s} A U^{s T}\right]^{-1}
$$

While $\mathbf{r}_{b}$ describes a change of $b m i$ by one unit with constant age and gender and with minimal shape variation, $\mathbf{r}_{b}$ and $\mathbf{r}_{b}$ alter age and gender correspondingly. The effects caused by adding these basis vectors to the average face in Fig. 1 (b) are shown in Fig. 2.

\section{Combined TPS-PCA-based Model Fitting}

Before applying the combined statistical and TPS reconstruction procedure, the set of given skull property values for a particular target skull is imposed as a hard constraint by eliminating variation in the statistical model coded by the given values and generating a property normalized statistical model. This is realized by subtracting the basis vectors $R$, from the previous section, weighted by the corresponding differences $\left(\triangle^{k} b m i, \triangle^{k}\right.$ age, $\triangle^{k}$ gender $)$ from the model description parameters $\mathbf{c}^{k}$ of each facial entry $\left\{F_{k} \mid k=1, \ldots, K\right\}$ in the database according to:

$$
\tilde{\mathbf{c}}^{k}=\mathbf{c}^{k}-R\left[\begin{array}{c}
\triangle^{k} \text { bmi } \\
\triangle^{k} \text { age } \\
\triangle^{k} \text { age }
\end{array}\right]
$$

As such, a new database is obtained which is invariant with respect to the given property values of the skull specimen. Every face in the database has now the same property values equal to the given values. The remaining variability in this property value-normalized population is smaller than the original population and can be calculated by applying a new PCA to the normalized set of instances $\tilde{\mathbf{c}}^{k}$. The result is a new property value-normalized statistical model $\tilde{M}(\mathbf{c})$.

A TPS-based reconstruction in combination with the new flexible statistical model is obtained by solving the following TPS interpolation problem. Given corresponding landmark points $\left(F_{i}^{l m}, \tilde{M}_{i}^{l m}(\mathbf{c})\right)$, determine a smooth model mapping or deformation function $\mathbf{f}$, with parameters $(\mathbf{d}, \boldsymbol{\Lambda})$, and model parameters $\mathbf{c}$, satisfying the following interpolation conditions: 


$$
F_{i}^{l m}=\mathbf{f}\left(\tilde{M}_{i}^{l m}(\mathbf{c})\right) \quad \text { for } \quad i=1, \ldots, L
$$

while minimizing the following reconstruction functional (using the notations of Eqs. (4) and (11):

$$
\begin{aligned}
E_{\mathrm{rec}}(\mathbf{c}, \mathbf{d}, \boldsymbol{\Lambda})= & E_{\mathbf{f}}+E_{\mathbf{f}(\tilde{M}(\mathbf{c})), \tilde{M}(\mathbf{c})}(\mathbf{c}) \\
= & \operatorname{trace}\left(\boldsymbol{\Lambda}^{T} \Phi \boldsymbol{\Lambda}\right)+ \\
& \|\mathbf{f}(\tilde{M}(\mathbf{c}))-\tilde{M}(\mathbf{c})\|^{2}+ \\
& \sum_{k=1}^{K-1}\left(\frac{c_{k}}{\sigma_{k}}\right)^{2}
\end{aligned}
$$

with $\Phi_{i l}=\left\|\tilde{M}_{i}^{l m}(\mathbf{c})-\tilde{M}_{l}^{l m}(\mathbf{c})\right\|$. The first term in the reconstruction energy functional is the same as in equation (4) regularizing the non-affine part of the TPS-deformation defined by interpolating the corresponding landmark points $\tilde{M}^{l m}(\mathbf{c})$ and $F^{l m}$. The second term minimizes the distance of the TPS-deformed complete model $\mathbf{f}(\tilde{M}(\mathbf{c}))=\mathbf{f}\left(\left(\tilde{M}^{s}(\mathbf{c}), \tilde{M}^{l m}(\mathbf{c})\right)\right)$ to the closest face model instance favoring facial templates with limited overall deformation, not just at the landmark points. The last regularization term favors more plausible face model parameters $\mathbf{c}$.

Solving the interpolation problem (18), while minimizing (19), is done iteratively by alternating the estimation of the model parameters (c) and the TPS parameters $(\mathbf{d}, \boldsymbol{\Lambda})$. When the model parameters are kept fixed, the interpolation problem is similar to the one defined in section 2 and the TPS parameters can be updated according to equation (5). A new estimate $F_{\text {est }}^{s}$ of the facial surface points $F^{s}$ can then be calculated using equation (6). Keeping the TPS parameters $(\mathbf{d}, \boldsymbol{\Lambda})$ fixed and based on the new facial estimate, the model parameters can be updated by maximizing the a posteriori probability in equation (10), where $F=\left(F_{\text {est }}^{s}, F^{l m}\right)$. For model initialization we can take the average face $\bar{F}$ by setting the model parameters $(\mathbf{c})$ equal to zero.

The complete iterative updating algorithm is described in pseudo-code below:

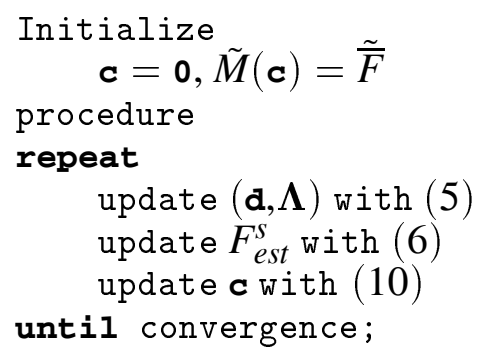

\section{Results}

Comparative reconstructions of a real-case skull found in Belgium are presented in this section to show the differences in the reconstruction models used (static versus statistical). In order to make the reconstructions comparable, all models used in this section have 52 skull landmarks and an estimate of the nose tip as additional skin landmark to determine the model deformation. A 3D digitized model of the skull, acquired with CT scanning and the 52 skull landmarks are depicted in Fig. 3. An estimate of the nose was set out according to [15].

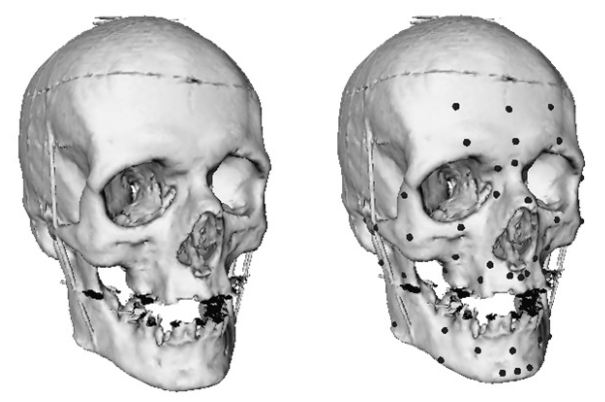

Fig. 3. 3D digitized model of the input skull (left) and the 52 skull landmarks indicated on the skull (right).

Two different static models were used to make a facial reconstruction of the skull in figure 3 based on the TPS deformation machinery explained in section 2 . The first is the average face of our database, being a generic facial model. The second is a subject-specific face from the database. The models and reconstruction results are shown in figure 4 . The model bias in the reconstructions is clearly visible. The result from the average face (figure 4 (b)) is very smooth and contains no specific facial characteristics, similar to the average face. When using the subject-specific facial template, facial characteristics of the template remain in the final reconstruction (the eyebrows and the chin region, e.g). Furthermore, large differences between several landmarks on the model and the target skull (the nose tip e.g) generate caricaturelike final outlooks of the reconstructions due to the large amount of non-affine deformation 


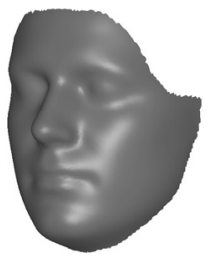

(a)

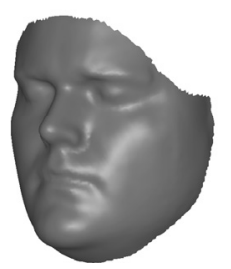

(c)

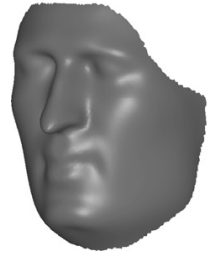

(b)

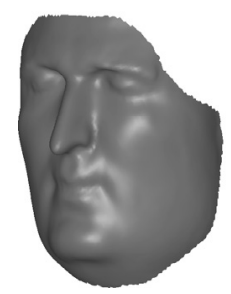

(d)
Fig. 4. Two static facial templates ((a) generic face, (c) specific face), and their TPS-based reconstruction results $(b, d)$.

that has been applied. Especially, when using the subject-specific face, the nose of the reconstruction (figure 4 (d)) looks unrealistic.

A facial reconstruction of the skull in figure 3 was also made with our proposed flexible statistical model, as explained in section 5. In the first test, the property values were set to: $b m i=20$ (thin person), age $=25$ and gender $=1$. Then the model was initialized with the average face of the property-normalized database. During reconstruction, the flexible model and the TPS deformation were iteratively updated, resulting in the facial template and the reconstruction depicted in figure 5 (a) and (b), respectively. In the second test, we used a subject-specific face of the normalized database as initialization for the model and we observed that the final template and reconstruction were exactly the same as in figure $5(\mathrm{a}, \mathrm{b})$. In fact, any face from the property-normalized database could have been used to initialize the model leading to the same template and reconstruction results. When observing the reconstruction in figure $5(\mathrm{~b})$, more characteristic facial features are apparent in the reconstruction than in the one based on the average model in figure 4 (b), but they cannot be attributed to a subject-specific face in the database, unlike the reconstruction based on the subject-specific face model in figure $4(\mathrm{~d})$. In

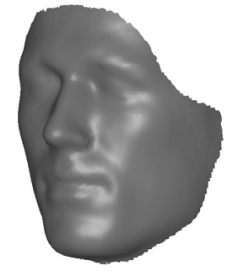

(a)

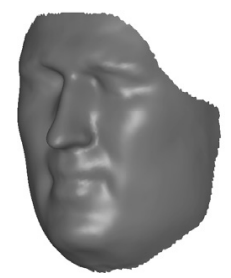

(c)

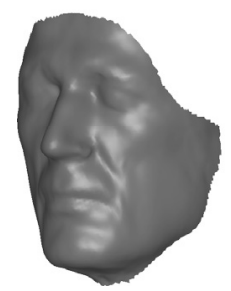

(e)

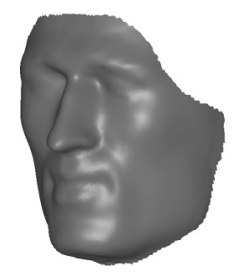

(b)

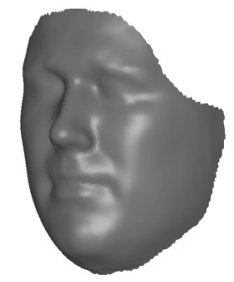

(d)

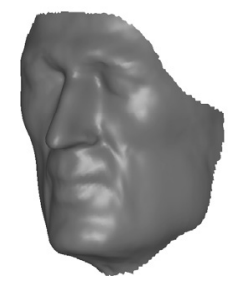

(f)
Fig. 5. Three different reconstructions of the skull in figure 3 based on a statistical model in combination with a TPS deformation $(\mathrm{a}, \mathrm{b})$ Template and reconstruction for $\mathrm{bmi}=20$, Age $=25$ and gender $+1 ;(\mathrm{c}, \mathrm{d})$ Template and reconstruction for bmi $=30$, Age $=25$ and gender +1 ; $(\mathrm{e}, \mathrm{f})$ Template and reconstruction for $\mathrm{bmi}=20$, Age $=70$ and gender +1 .

other words, no bias towards a specific face in the database is introduced into the reconstruction. Finally, the plausibility of the reconstruction being a human face in figure 5 (b) is much higher than the caricatural ones in figure $4(\mathrm{~b}, \mathrm{~d})$, especially when observing the nose. This can be explained by the fact that the differences between the model landmarks and the skull landmarks are reduced during reconstruction by applying face-specific variations so that a smaller amount of TPS deformation is needed. Indeed, the difference between the final statistical model template and the final reconstruction is much smaller than the differences between templates and reconstructions in 4 . (a,c) and $(\mathrm{b}, \mathrm{d})$, respectively.

Two more reconstructions were made based on different property values to illustrate the possibilities of the proposed reconstruction method. 
When a different set of property values is suggested, manual reconstructions by means of modeling plasticine need to be completely redone, while reconstruction methods based on a static model require a new facial template with the proper properties to start from. With our proposed method, no new template is required and a new facial reconstruction is made within a few seconds. In figures 5 (c) and (d) the facial template and reconstruction for an increase of bmi (30) are shown, while figures 5 (e) and (f) show the results for an increase in age (70). When observing the different templates and reconstructions in figure 5 , one can see that the major facial characteristics remain visible over the different reconstructions, while the property-specific characteristics are clearly different. This is because the set of basis vectors describing property-dependent characteristics, model a change in property with minimal shape variation.

\section{Conclusion}

We proposed a flexible statistical facial model of combined tissue-depths and complete facial surfaces, which can be used for 3D computerized forensic facial reconstructions. The main difference with currently used facial models is the automatic adjustment or improvement of the model by making use of face-specific modes of variation, which in combination with a TPS-based interpolation results in unbiased and more realistic reconstructions. To adjust the statistical facial model parameters we augmented the TPS minimal bending energy interpolation functional with a face-specific regularization term. Minimizing the augmented energy functional was done iteratively by alternating the estimation of the PCA-based statistical model parameters and the TPS deformation parameters until convergence. Before the iterative reconstruction procedure, property values of a given skull specimen were imposed as hard constraints by removing the variability in the database and the statistical model coded by differences in property values. As a result, multiple reconstructions of the same skull, but with different property conditions, can be made within a few seconds. A comparison was made between a reconstruction with the proposed model and reconstructions with a generic and a subject-specific static facial model on a realcase skull. The result of this approach might be interpreted as an improvement in terms of facial plausibility of the reconstruction. Different initializations of the model were used, leading to the same template and reconstruction results, which indicate that no bias towards a specific face was introduced into the reconstruction. Finally, multiple reconstructions were made in order to illustrate the possibilities of the proposed statistical method for alternating the skull property values. Some extensions can be proposed to the reconstruction based on a flexible model in combination with a TPS deformation. First of all, having a larger and more diverse database increases the flexibility of the model. The more the model can be adjusted towards the skull in a face-specific way, the smaller the amount of TPS deformation required. Ideally, no more extra TPS deformation is needed in order to satisfy the interpolation conditions. The current database of 118 faces is rather limited and will be expanded in the future. Secondly, the indication of the skull landmarks is currently done manually and is error-prone. It would be interesting to relax the interpolation conditions during TPS fitting according to error ranges on the landmark indications. Another solution could be the automatic extraction of the skull landmarks from the skull, such that the indication would be less error-prone and such that the complete reconstruction pipeline would be automatic. Finally, a proper validation scheme for cranio-facial reconstructions is to be defined and applied in order to evaluate and compare the performance of different reconstruction methods. The goal of cranio-facial reconstruction is recognition or identification. However, these concepts are hard to translate into a proper and easy validation scheme.

\section{References}

[1] P. VANEZIS, Application of 3-D computer graphics for facial reconstruction and comparison with sculpting techniques, Forensic Science International, vol. 42, pp. 69-84, 1989.

[2] P. Vanezis, M. Vanezis, G. McCombe and T. NIBLET, Facial reconstruction using 3-D computer graphics, Forensic Science International, vol. 108, pp. 81-95, 2000. 
[3] R. Evenhouse, M. Rasmussen and L. SAdLer, Computer-aided Forensic facial reconstruction, Journal of BioCommunication, vol. 19(2), pp. 2228, 1992.

[4] A.W. Shahrom, P. VANezis, R.C. Chapman, A. GonZALes, C. Blenkinsop, M.L. Rossi, Techniques in facial identification: computer-aided facial reconstruction using laser scanner and video superimposition, Int. J. Legal Med, vol. 108, pp. 194-200, 1996.

[5] A.J. Tyrell, M.P. Evison, A.T. Chamberlain AND M.A. GREEN, Forensic three-dimensional facial reconstruction: historical review and contemporary developments, Journal of Forensic Science, vol. 42(4), pp. 653-661, 1997.

[6] G. QUATREHOMME, A fully three-dimensional method for facial reconstruction based on deformable models, Journal of Forensic Science, vol. 42(2), pp. 649-652, 1997.

[7] L.A. NeLson AND S.D. Michael, The application of volume deformation to three-dimensional facia reconstruction: a comparison with previous techniques, Forensic Science International, vol. 94, pp. 167-181, 1998.

[8] M.W. JONES, Facial reconstruction using volumetric data, Proceedings of the 6th International Vision Modeling and Visualization Conference - VMV-01, Stutgart, November 21-23, 2001., pp. 135-150.

[9] H. Chui and A. Rangarajan, A New Algorithm for Non-Rigid Point Matching, IEEE Conference on Computer Vision and Pattern Recognition (CVPR), vol. 2, pp. 44-51, 2000

[10] E. HuOt, H. Yahia, I. COHEN AND I. Herlin, Matching Structures by Computing Minimal Paths on a Manifold, INRIA, Air Project France, Universaty of Southern California, USA.

[11] T.F. COOTES AND C.J. TAYLOR, Statistical Models of Appearance for Computer Vision, Technical Report, October 2001

[12] J. Hug, C. BREChBuHLER AND G. SZEKELY, Tamed Snake: A Particle System for Robust SemiAutomatic Segmentation, Technical Report, BIWITR-185, March 1999

[13] S. De Greef, P. Claes, Dirk Vandermeulen et al., Semi-automated ultrasound facial soft tissue depth registration: method and validation, Journal offorensic sciences, in press, 2005.

[14] J. Muller, A. MANG AND T.M. Buzug, A Template-Deformation Method for Facial Reproduction, Proceedings of the 4th Int. Symposium on Image and Signal Processing and Analysis, September 15-17, pp. 359-364, 2005.

[15] K.T. TAYLOR, Forensic art and illustration, $C R C$ Press New York, 2001.
Received: December, 2005

Accepted: February, 2006

Contact address: Peter Claes

Katholieke Universiteit Leuven Medical Image Computing - ESAT/PSI U.Z. Gasthuisberg, Herestraat 49 3000 Leuven, Belgium e-mail: pclaes@uz.kuleuven.ac.be

PETER ClaES is currently doing a Ph.D. at the department ESAT/PSI/ Medical Image Computing of the K.U.Leuven (Belgium). The main topic of his research is surface-based cranio-facial statistical modeling and reconstruction. He obtained his M.Sc. in Engineering (ElectroTechnical) in 2002 from the same university. Before that he had done three projects as a student job concerning software development at Siemens Mobile in Herentals (Belgium).

DIRK VANDERMEULEN received a M.Sc. in engineering (computer science) from the K.U.Leuven (1983) and obtained his Ph.D. in Computer Science from the same university in 1991. He is currently associate professor at the department ESAT/PSI (Center for Processing Speech and Images) of the K.U.Leuven and member of the Medical Image Computing (ESAT/PSI + Radiology) research group at the same university. His research focuses on biomedical image analysis and biometric systems with a particular emphasis on image registration and object modeling.

SVEN DE GREEF cpmpleted his dental studies in 1995 at the Vrije Universiteit Brussel in Belgium. He is a member of the Belgian Disaster Victim Identification team. Also being an active Red Cross volunteer for several years, he followed a post-academic formation in disaster management and medicine at the Katholieke Universiteit Leuven (Belgium) after which he obtained the degree of a legal expert at the Rijks Universiteit Gent (Belgium). He is currently doing a $\mathrm{PhD}$ thesis at the department Forensic Odontology (Katholieke Universiteit Leuven). The main topic of his research is facial soft tissue depths and parts of the European Caucasoid. Since 2004 he has been a member of the International Association of Cranio-facial Identification.

GUY WILLEMS completed his dental studies in 1988 at the Katholieke Universiteit Leuven in Belgium. In 1992 he successfully defended his $\mathrm{Ph} . \mathrm{D}$. thesis at the same university. Related to his clinical training he received the Certificate of postgraduate training in operative dentistry with a special emphasis on aesthetic dentistry in 1993 and the Certificate of postgraduate training in orthodontics in 1996, both from the Katholieke Universiteit Leuven. Since then he has been enrolled for a tenure track position at the Department of Orthodontics, School of Dentistry, Oral Pathology and Maxillo-facial Surgery, Faculty of Medicine of the Katholieke Universiteit Leuven, Belgium (www.mfo.be). Professor Guy Wilems is a forensic odontologist at the Centre of Forensic Medicine, Katholieke Universiteit Leuven. He is founder of the Centre of Forensic Odontology at the School of Dentistry, Oral Pathology and Maxillofacial Surgery, he teaches forensic odontology at undergraduate and post graduate level and is coordinator of the international Master in Forensic Odontology (www.mfo.be).

PAUL SuETENS received a M.Sc. in engineering (computer science) from the K.U.Leuven (1977) and obtained his Ph.D. in Computer Science from the same university in 1983. He is currently a professor and head of ESAT/PSI (Center for Processing Speech and Images) and of the laboratory for Medical Image Computing (ESAT/PSI + Radiology) at the K.U.Leuven. His research focuses on medical imaging and medical applications of computer vision, 3D visualization, cranio-facial surgery and reconstruction, multimedia and telecommunication. 